\title{
Distribution Pattern of Nonmetallic Inclusions on a Cross Section of Continuous- Cast Steel Billets For Rails
}

\author{
Alexander A. Kazakov ${ }^{1}$, Andrey Zhitenev ${ }^{1}$, Pavel Kovalev ${ }^{1}$ \\ 1. St.Petersburg State Polytechnical University/Metallurgical Technologies Department, St.Petersburg, \\ Russian Federation
}

Nonmetallic inclusions are formed at all stages of the steelmaking process of melting and secondary treatment before casting and crystallization. Redistribution of impurities and alloying elements between solid and liquid phases during crystallization occurs [1], forming chemical and structural inhomogeneity on a cross section of the billet. The formation of inclusions in a solidifying steel occurs simulteniously with a formation of a dendritic structure. All of these interconnected processes determine a distribution pattern of non-metallic inclusions in a billet's cross section [2].

Dendritic structure was revealed by hot etching in $50 \%$ water solution of $\mathrm{HCl}$ for 20 min. After this a specimen was swabbed in nital to remove a sludge and immersed by $\mathrm{cc} . \mathrm{H}_{3} \mathrm{PO}_{4}$ for blackening a surface. Next the sample was polished by abrasive paper P1200 to remove blackening from dendrite arms.

For a quantitative determination of a dendritic structure the secondary dendritic arm spaces (SDAS) were evaluated. To obtain reliable results panoramic images of a dendritic structure were created by means of Image Analyzer ThixometPro ${ }^{\mathrm{TM}}$. An estimation of nonmetallic inclusions were performed in accordance with the standard ASTM E 1245.

Fig. 1 shows an panoramic image of an identified dendritic structure. All structural zones formed during crystallization [1] were distinguished: outer equiaxed zone, columnar zone, inner equiaxed zone. Columnar crystals zone are more extended from a side of the inner radius of continuous casting machine because of a greater cooling rate.

The distribution pattern of nonmetallic inclusions on the cross section of continuous-cast steel billets for rails in consideration of dendritic parameters in all crystal structure zones were studied. Maximum nonmetallic impurity rating was detected at the boundary intersection of adjacent crystal structure zones.

The secondary dendrite arm spacing SDAS $\left(\lambda_{2}\right)$, which characterizes the size of dendritic cell, was examined on the cross section of a billet. For the rail continuous-cast steel billet there is $\lambda_{2}$ increase only for the columnar crystal zone while for the equiaxed zone it is unaffected (fig.1). Local areas with increased SDAS exist due to maximum solidification local time. Areas of local thermal centers are located irregularly and asymmetrically with respect to the geometric center of the billet.

It was that determined effects of secondary dendrite arm spacing upon the dimentions of tertiary nonmetallic inclusions appeared during solidification. That is for rail continuous-cast steel billet such a relationship was found only for a columnar crystal zone when in the region of equiaxed zone a size of sulfides varies stochastically (fig.2) due to the macrosegregation phenomena.

Method of sampling for the control of nonmetallic inclusions in continuous-cast steel billets for rails was developed [2] based upon revealed relationships between nonmetallic inclusions formation and dendritic structure formation which correlates good with a sampling for end products (rails). 


\section{References:}

[1] Kurz W., Fisher D.J. Fundamentals of solidification. - Trans. Tech. Publ., 1998.-305 p.

[2] Kazakov A.A., et al., Chernye Metally (Ferrous Metalls), 2014, №4 (988), p. 31.

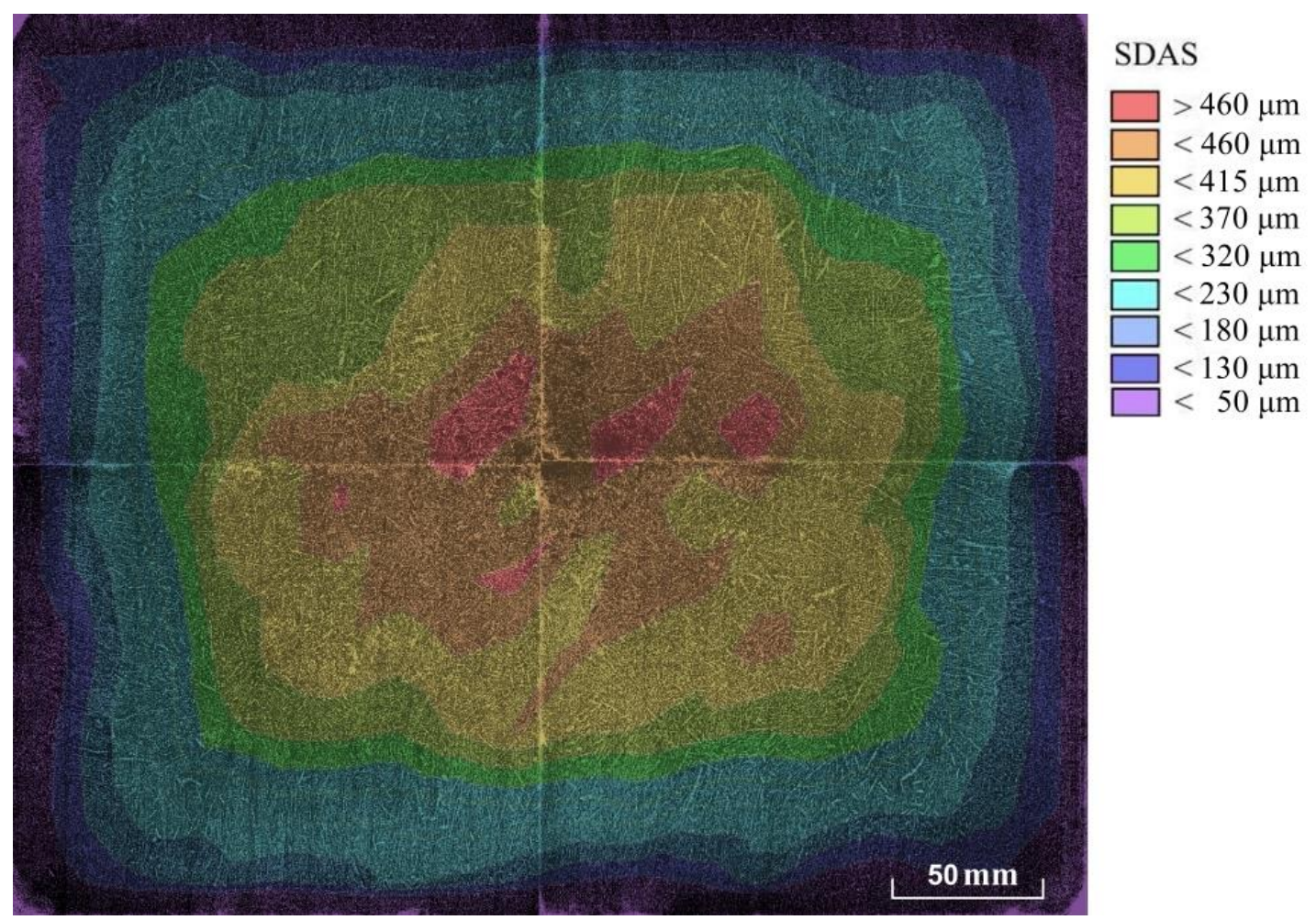

Figure 1. Macrostructure of a billet's specimen and measured distribution of SDAS on a cross section of the billet

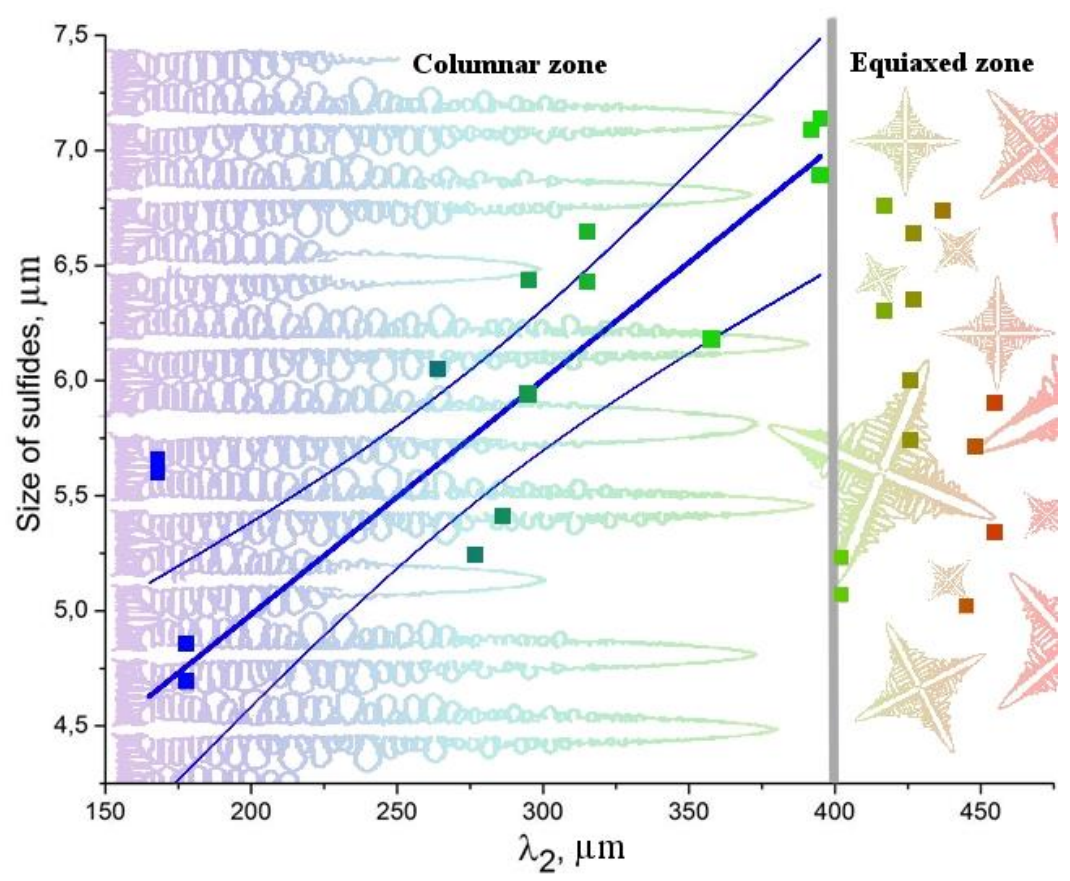

Figure 2. Relationship between sulfide's size and secondary dendrite arm spaces 